\title{
Experimental observation of spontaneous spin polarization of electrons in hybridized states of transition element impurities in semiconductors
}

\author{
V. I. Okulov, ${ }^{\text {a) }}$ T. E. Govorkova, I. V. Zhevstovskikh, A. T. Lonchakov, and K. A. Okulova \\ Institute of Metals Physics, Urals Branch, Russian Academy of Sciences, ul. S. Kovalevskoi 18, \\ Ekaterinburg 620990, Russia
}

\author{
E. A. Pamyatnykh \\ Urals Federal University, pr. Lenina 51, Ekaterinburg 620083, Russia
}

\section{S. M. Podgornykh}

Institute of Metals Physics, Urals Branch, Russian Academy of Sciences, ul. S. Kovalevskoi 18, Ekaterinburg 620990, Russia

M. D. Andriichuk

Urals Federal University, pr. Lenina 51, Ekaterinburg 620083, Russia

\section{D. Paranchich}

Chernovtsy National University, Chernovtsy, Ukraine

(Submitted December 11, 2012)

Fiz. Nizk. Temp. 39, 493-498 (April 2013)

Experimental evidence of the possible existence of spontaneous spin polarization of the electron system in hybridized states formed by transition element impurity atoms in the conduction band of semiconducting crystals is examined. The details of a quantitative interpretation of experiments on the temperature dependence of the specific heat and elastic moduli of mercury selenide crystals with iron impurities confirm the feasibility of establishing the presence of electron spin polarization in this type of experiment, as well as the possible existence of polarization in the crystals studied here. Theoretical arguments support the observation of a thermodynamic anomalous Hall effect owing to spontaneously polarized donor electrons from low-concentration impurities. (C) 2013 AIP Publishing LLC [http://dx.doi.org/10.1063/1.4801435]

\section{Introduction}

The physical properties of semiconductors with transition element impurities have long been of interest for many reasons. Recently this interest has focussed mainly on problems relating to the occurrence of spontaneous spin polarization of conduction electrons at room temperature in sufficiently perfect systems with a well controlled structure. The customary way of solving these problems is to create ever more complicated multicomponent systems. Based on our studies, we hope to demonstrate the effectiveness of yet another way in which systems with hybridized impurity electron states are used. Hybridization of the electronic states in the conduction band of a crystal matrix is a quite widespread phenomenon that is typical, in particular, of narrow gap crystals with transition $d$-element impurities. We have done ${ }^{1}$ experiments on mercury selenide crystals with impurities of the $3 d$-elements and provided a detailed quantitative interpretation of the experimental data that yielded a reliable determination of the parameters of the hybridized states. The electron density in the hybridized states corresponds to a combination of localization and free motion. Localization in the conduction band can produce an effective spontaneous spin polarization of the electrons in the hybridized states. Then the conducting part of the states ends up polarized, which can mean that the conduction electrons can be polarized at high temperatures. In a theoretical description of this scheme $^{2}$ it was pointed out that previous data on the temperature dependences of the specific heat ${ }^{3}$ and sound speed ${ }^{4}$ may correspond to the existence of spontaneous spin polarization of the electrons in hybridized states in mercury selenide with iron impurities. The present article can be regarded as an extension of Ref. 2. Here we give a more detailed justification of the analysis of the experimental data $^{3,4}$ and then consider a theoretical argument for the feasibility of direct observation of spontaneous spin polarization of conduction electrons in experiments on the anomalous Hall effect.

\section{Detecting spontaneous polarization of hybridized states in impurity electron thermodynamic effects}

It is difficult to detect spontaneous polarization of electrons in the objects considered here because of the small magnitude of this polarization. Nevertheless, proof of the existence of this effect is of great interest, both because of its fundamental significance, and because of the high likelihood of technological applications. As a preliminary argument in favor of further study, we note that, according to data on magnetic susceptibility, the states of the electrons in the transition element impurity atoms, which become donors in the conduction band, have an initial spin polarization. In particular, the state of one electron of an iron impurity atom in the conduction band of crystalline mercury selenide has a spin 
polarization opposite in sign to the coexisting polarization of the states of the five electrons in the valence band. This spin polarization effect is caused by intra-atomic exchange interactions corresponding to energy on an atomic scale. Of course, the scale of the interaction energy, which provides for this polarization of the aggregate of impurity donor electrons in the conduction band of the crystal, can be much smaller, since this is facilitated by the spreading of the electron density over the entire crystal. However, hybridization of the states of the localized (atomic) and free motion can reduce the role of this spreading in the weakening of the exchange interaction, since it the localization then contributes to the electron density. Thus, hybridization means that it is possible, to some extent, to conserve the original polarization of the donor electron for the entire aggregate of these electrons. This idea has raised the possibility of spin polarization of the electron systems in hybridized states and of an experimental observation of this effect. The theoretical problem has been examined previously ${ }^{2,5}$ and here we discuss the problem of observing this effect experimentally.

The experimental data are interpreted using the previously developed description of hybridized states, for which the basic foundations were discussed in detail in Ref. 2. Spontaneous spin polarization of these kinds of states under the assumption that the entire electron system has the same sign for the polarization within a single hybridization interval is modelled by introducing a shift in the energies of the electrons within this interval by an negative polarization energy $\beta . \beta$ is given by

$$
\beta=\psi \int d \varepsilon n_{i} \omega_{i}(\varepsilon) f(\varepsilon+\beta / 2)
$$

where $\psi$ is an energy parameter characterizing the interelectron exchange interaction, $n_{i}$ is the impurity concentration, which equals that of the donor electrons, and $f(\varepsilon)=\{\exp [(\varepsilon-\varsigma) / T]+1\}^{-1}$. This equation only includes the part of the density of electron states related to the localized electron density and is taken as the basis for determining the spin polarization. In our approach, it is described by the function

$$
\begin{aligned}
\omega_{i}(\varepsilon)= & (\Delta / \pi)\left\{\left[\left(\varepsilon-\varepsilon_{r}\right)^{2}+\Delta^{2}\right]^{-1}\right. \\
& +(1 / \Gamma \Delta)[(\pi / 2-\arctan (\Gamma / \Delta)]\},
\end{aligned}
$$

which is nonzero within the hybridization interval $\varepsilon_{r}-\Gamma<$ $\varepsilon<\varepsilon_{r}+\Gamma$ near the resonance energy $\varepsilon_{r}$ and has a peak of width $2 \Delta$. The chemical potential $\varsigma$ is derived from the condition that the total number of occupied electronic states per unit volume is equal to $n_{i}$, i.e.,

$$
\int d \varepsilon n_{i} \omega_{i}(\varepsilon) f(\varepsilon+\beta / 2)=n_{i}-n_{e},
$$

where $n_{e}$ is the concentration of the free-motion component (conduction electrons) in the hybridized states given by the electron concentration of the uncontrolled donors. The energy dependence of $n_{e}$ within the resonance interval is assumed to be insignificant, and we take $n_{e}(\varepsilon) \approx n_{e}\left(\varepsilon_{r}\right)$ $\equiv n_{e}$. Equations (1) and (3) then yield a simple expression for the energy $\beta$

$$
\beta=\psi\left(n_{i}-n_{e}\right)
$$

This expression applies when the initial assumption about a single sign for the polarization of all the electrons, which reduces to the condition $|\beta|>2 \Gamma$. It is useful to rewrite this inequality in the form proposed in Ref. 1 by introducing the dimensionless parameter $A=\psi g_{c}\left(\varepsilon_{r}\right)$ in place of the energy $\psi$, where $g_{c}\left(\varepsilon_{r}\right)$ is the density of states of the conduction band of the crystal in the hybridization interval for the impurity states. It is also useful to introduce the energy parameter $E_{r}=n_{e} / g_{c}\left(\varepsilon_{r}\right)$ which characterizes the scale of the variations in the density of states of the conduction band within the hybridization interval. Then the condition for the existence of spontaneous spin polarization of the hybridized states can be written in the form:

$$
\left(n_{i} / n_{e}\right)|A|>\left(\Gamma / E_{r}\right) .
$$

The parameter $A$ is analogous to the standard parameters of the electron Fermi-liquid interaction, so that, in terms of order of magnitude, it is typically close to unity. The parameter $\left(\Gamma / E_{r}\right)$ is small compared to unity, so that in evaluating the realism of the inequality (5) there is reason to assume that spontaneous spin polarization of the system of electrons in the hybridized states is entirely possible. The polarized system includes polarized conduction electrons belonging to it and their polarization energy $\beta_{c}$ is given by

$$
\beta_{c}=\left(\psi_{i c} / \psi\right) \beta=\psi_{i c}\left(n_{i}-n_{e}\right),
$$

where $\psi_{i c}$ is a parameter characterizing the exchange interaction between the components of different states.

The condition (5) for attainment of polarization turns out to be important for detecting the polarization effect, since direct measurement of $\beta$ is difficult because it is so small. (A method for this kind of measurement is discussed in the next section.) If $A$ is determined from the experimental data, then it can be used to establish whether spin polarization of the electron system occurs. In our papers on the temperature dependences of the impurity contributions to the specific heat and the moduli of elasticity of crystalline mercury selenide crystals, ${ }^{3,4}$ a detailed quantitative interpretation of the data has been used to determine the parameters (including $A$ ) of the hybridized states. This result, which presumably corresponds to the existence of spontaneous spin polarization of the hybridized states, has been discussed in Ref. 2. Here we briefly summarize and illustrate the results of a more detailed interpretation of the experiments ${ }^{3,4}$ from the standpoint of its reliability in the aspect discussed here. This must be done for the natural reason that these experiments were done and interpreted without considering the possibility of spin polarization of the states and it was not clear at that time whether clear data on hybridized states could be obtained at all in these experiments. Ultimately, it turned out that the observed temperature dependences of the impurity contributions to the specific heat and the slow transverse sound speed have nonmonotonic variations (a maximum in the specific heat and a minimum in the sound speed) that are well described by the formulas given in our papers relating these characteristic variations to the appearance of hybridized impurity electronic states. The additional analysis carried out here has shown that these formulas are also fully 
applicable to the case of spin polarization described in the models described above. Here the contribution of the interelectron exchange interaction is taken into account with the same simplified model, with this interaction characterized by the same parameter $A$. Its influence on the temperature dependences of the impurity contributions to the thermodynamic quantities is described by the factor $(1+u \eta)^{-1}$, where

$$
u=\left(n_{i} / n_{e}\right)(1+A), \quad \eta=\operatorname{Er} \int d \varepsilon[-\partial f(\varepsilon) / \partial \varepsilon] \omega_{i}(\varepsilon),
$$

The high sensitivity of the form of the observed temperature extremes to the magnitude and, especially, the sign of the quantity $u$ that was found during fitting of the theoretical curves to the experimental data is very important for solving this problem. This behavior is illustrated in Figs. 1 and 2. The examples plotted in these figures illustrate the results of a detailed analysis that yielded two facts: the impossibility of obtaining a satisfactory match between the theoretical curve and the experimental data for positive $u$ and the large changes in the theoretical curves for small (less than a few tenths) variations in $u$. Good fits for the specific heat and the sound speed were obtained with the same negative value of $A$ with an absolute value well above unity for the corresponding impurity concentrations. It is easy to understand the significance of these results. If $u<0$, this means that $A$ is negative (a necessary condition for spin polarization) and has a modulus greater than unity (the inequality (5) is satisfied). Thus, the supplemental points raised in this paper provide serious arguments in favor of a substantial intensity of the interelectron exchange interaction in the electron system of the hybridized states that would be sufficient to produce spin polarization. But this conclusion is based on studies of temperature anomalies in the thermodynamic properties that are not directly related to spin polarization and are also limited by the simplifications in the models for a quantitative theoretical description of both the measured quantities and of spin polarization. Further progress in detecting and studying spin polarization in the objects we have studied will, of course, follow from experiments for observing it directly.

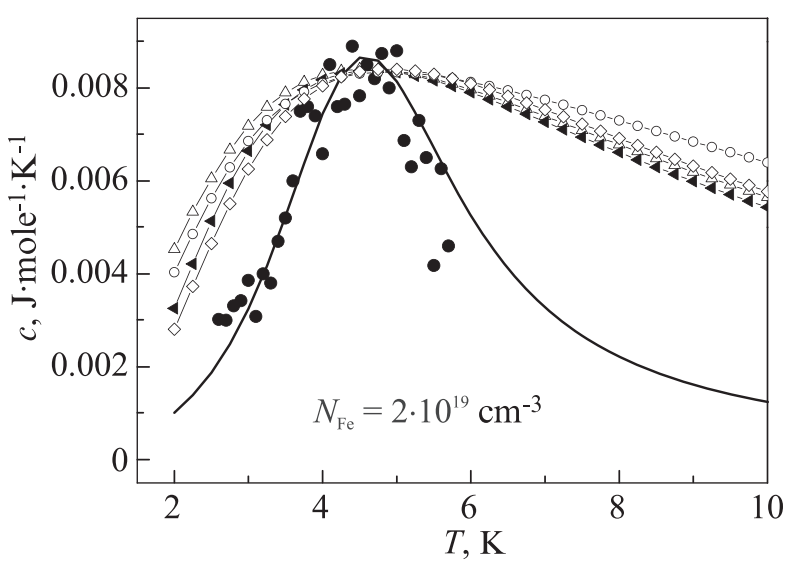

FIG. 1. Temperature dependence of the impurity part of the specific heat in HgSe:Fe crystals with $N_{\mathrm{Fe}}=2 \times 10^{19} \mathrm{~cm}^{-3}$ for different values of the Fermi-liquid interaction parameter $A(u=1+A)$. The smooth curve corresponds to $u=-0.22$ and is the best fit to the experimental data indicated by the points $(\bullet)$; also shown are the curves for $u=0(\triangle), 0.15(\checkmark), 0.3(\bigcirc)$, $0.22(\diamond)$.

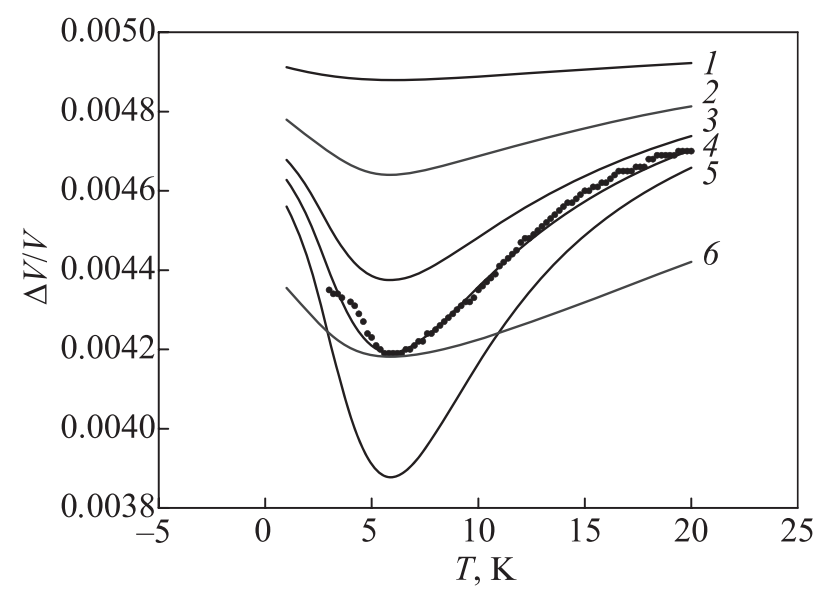

FIG. 2. Temperature dependence of the phase velocity of the slow transverse wave measured at a frequency of $54 \mathrm{MHz}$ in a sample of $\mathrm{HgSe}: \mathrm{Fe}$ with a concentration $\mathrm{Fe}=1 \times 10^{19} \mathrm{~cm}^{-3}$. The smooth curves are calculated for the following values of $A$ : $A-0.5(1)-1(2),-1.15(3),-1.2(4)$, $-1.25(5), 1.2(6)$. It was not possible to obtain good agreement with the experimental curve for positive values of $A$, even when the other fit parameters were varied.

The thermodynamically anomalous Hall effect and the possibility of using it for studying spin polarization of the electron systems in hybridized states

The anomalous Hall effect, in which spontaneous magnetization makes a characteristic contribution to the Hall resistance, is widely used to study magnetic ordering in conducting crystals. Recently, there has been a rapid increase in interest in this effect, especially in the physics of semiconductors and low-dimensional structures doped with transition element impurities. Research in this area has been reviewed in Refs. 6 and 7. This section of this paper is devoted to a theoretical justification of the possibility of observing spontaneous spin polarization in experiments on a special type of anomalous Hall effect.

The standard theories attribute the anomalous Hall effect to electron spin-orbital interactions and mainly apply to objects with strong magnetism. The predicted magnitude of the effect in our systems with a small spin polarization would most likely be too small and would be hard to observe. In a search for other possibilities, we have examined another type of effect that is unrelated to spin-orbital interactions but arises from a thermodynamic, nondissipative aspect of the physics of the Hall effect. This aspect has obviously not been considered fully in the existing theories, so the thermodynamic type of anomaly has not been drawn much attention.

For the following discussion to be sufficiently rigorous and simple, it is appropriate first to define the concepts of the magnetization density vector and the magnetization current. In an initial homogeneous, unbounded electron system with spontaneous spin polarization, spontaneous magnetization develops as a result of the appearance of a magnetization current that can only occur as a result of inhomogeneity in the electron density. In a thermodynamic approach, this kind of inhomogeneity is described by a position dependence of the chemical potential $\varsigma$, which can, in this case, be identified with the electrochemical potential. The first and most important step in the transition from a homogeneous to an inhomogeneous system is to take the boundaries of the object into account. Let a spontaneous magnetization $M_{0} \mathbf{b}$, directed 
along the unit vector $\mathbf{b}$ and the axis of symmetry of the object, develop. Then the magnetization currents are concentrated near the contours of the cross sections of the boundaries of the object in planes perpendicular to the vector $\mathbf{b}$ in accordance with the distribution of the gradient of the chemical potential $\varsigma(\Gamma)$ along these closed contours. For the system we are considering, the magnetization current density is $\mathbf{j}_{\mathbf{m}}=c$ curl $M_{0} \mathbf{b}$. Then we find the average magnitude of the spin part of the current density for an electron with a Fermi function having chemical potential $\varsigma(\mathbf{r})$ to be

$$
\begin{aligned}
\mathbf{j}_{\mathbf{m}} & =\mu_{0} c \operatorname{curl}\left\{\int d \varepsilon g_{c}(\varepsilon) f(\varepsilon+\beta / 2) \mathbf{b}\right\} \\
& =-c\left(\partial M_{0} / \partial \varsigma\right)[\mathbf{b} \times \operatorname{grad} \varsigma(\mathbf{r})] .
\end{aligned}
$$

Equation (8) then determines the magnitude of the spontaneous magnetization

$$
M_{0}=\mu_{0} \int d \varepsilon g_{c}(\varepsilon) f(\varepsilon+\beta / 2)
$$

and the effective magnetization conductivity

$$
\sigma_{m}=e c\left(\partial M_{0} / \partial \varsigma\right)
$$

All these quantities are determined for the conducting component of the hybridized states, in accordance with the following discussion of the Hall effect.

The next step in describing the magnetization involves extending the above ideas to a system in an external magnetic field. The magnetic field leads to a realignment of the magnetization currents and the appearance of an induced magnetization that is proportional to the field strength (or has a more complicated field dependence). The energy of the additional spin polarization induced by the magnetic field is given by Eq. (1) with an additional inhomogeneous term proportional to the field strength. In a system with spontaneous spin polarization, there is a constant part of the magnetization that does not depend on the field strength. In a sufficiently high magnetic field (on reaching saturation) the magnetization $M \mathbf{h}$ in our system is oriented along the direction of the magnetic field and separates into two components, i.e.,

$$
M=M_{0}+m,
$$

where $M_{0}$ is independent of the field strength and is associated with the spontaneous polarization, while $m$ (the average magnetic moment of the electrons) corresponds to the contribution induced by the magnetic field.

Next, it is appropriate to introduce the thermodynamic potential of the electron system in the variables $\varsigma, T$, and $H-$ the pressure $P$. The derivative of the pressure with respect to the magnetic field strength equals the magnetization $M$, and it reproduces Eq. (11) since $P$ is the sum of two terms

$$
P=P_{0}+M_{0} H
$$

where $P_{0}$ is an even function of the magnetic field strength, the derivative of which gives $m$. For a more exact treatment, the field strength must be replaced by the magnetic induction, but this refinement is unnecessary here since the magnetization is so small.
We now consider the Hall effect. Let an electric field with potential $\varphi(r)$ and an emf, which induces a dissipative, as well as a nondissipative Hall current, be applied perpendicular to the magnetic field. The Hall conductivity is calculated using the quantum mechanical kinetic equation for the density matrix of the electrons. It is solved by introducing a locally equilibrium density matrix of a state which is described in a manner analogous to that used above for the magnetization currents. The formal difference lies in the replacement of the inhomogeneous chemical potential by the energy $e \varphi(r)$; the physical difference is more profound, although it does not prohibit the use of this analogy. In this regard, the analysis of the current in the locally equilibrium state must include the magnetization currents that arise in this state. The current density in the locally equilibrium is calculated in the same way as for the electrons in a quantizing magnetic field ${ }^{8,9}$ and is given by

$$
\mathbf{j}^{L}=c \operatorname{curl} n^{\varphi} \mathbf{h},
$$

where $m^{\varphi}$ is a quantity analogous to $m$ with the above substitution. Now we have to take into account the fact that an electric field with an emf differs fundamentally in that it leads to a conduction current which is the observed Hall current. A thermodynamic determination of the conduction current density $\mathbf{j}_{c}^{L}$ in a locally equilibrium state by the method proposed in Ref. 8 is based on the condition of equilibrium between the nondissipative thermodynamic forces of the pressure and the magnetic field acting on a conductor with a current, i.e., $\operatorname{grad} P=(1 / c)\left[\mathbf{j}_{c}^{L} \times \mathbf{H}\right]$. This yields

$$
\mathbf{j}_{c}^{L}=-(c / H)\left[\operatorname{grad} P^{\varphi} \times \mathbf{h}\right]=\left\{\left(e c n_{e} / H\right)+\sigma_{m}\right\}[\mathbf{E} \times \mathbf{h}] .
$$

After this, using Eq. (12) in Eq. (13) gives

$$
\mathbf{j}^{L}=c \operatorname{curl} \mathbf{M}+\mathbf{j}_{c}^{L},
$$

where

$$
\mathbf{M}=M_{0} \mathbf{h}+m \mathbf{h}+(1 / H) P_{0} \mathbf{h} .
$$

Equation (16) for the magnetization is consistent with the above idea of magnetization and spontaneous magnetization currents, while the last two terms (Landau diamagnetism and Pauli paramagnetism) are well known. Thus, we can regard the separating out of the conduction current by Eq. (15) as well justified. This means that the formula for the collisionless Hall conductivity in Eq. (14), which contains an anomalous contribution of magnetization conductivity (Eq. (10)), is also valid. Ultimately, we have established the possibility of a thermodynamically equilibrium Hall effect that is unrelated either to spin-orbital interactions or to the complicated details of scattering processes and the electronic states. The parameter of the anomaly is the conductivity $\sigma_{m}$ characterizing the magnetization currents. If we write it in the form $\sigma_{m}=e c n_{e} / H_{a}$, then for the conduction electron system we are discussing, the field strength $H_{a}$ is of an order of magnitude close to $M_{0}$. On estimating the Hall resistance $\rho_{H}$ using a Drude model under the condition $\rho \sigma_{m} \ll 1$, where $\rho$ is the electrical resistivity, we obtain

$$
\rho_{H}=\rho\left(\rho \sigma_{m}+\Omega \tau\right),
$$


where $\Omega \tau$ is the product of the cyclotron frequency and the electron relaxation time.

Estimates using Eq. (17) show that an anomalous contribution to the Hall resistance in the systems of electron hybridized states studied here can be observed with an attainable accuracy of measurement. This has been confirmed by room-temperature experiments on mercury selenide with iron impurities. ${ }^{10}$ Thus, there is reason to assume that the predicted spontaneous spin polarization does exist and the above interpretation of the anomalous Hall effect discussed here may serve as a basis for further research.

This work was supported by the Russian Foundation for Basic Research (Grants Nos. 11-02-90410 Ukr_f_a and 1202-00530) and Grant No. 12-T-2-1016 of the Division of Physical Sciences of the Russian Academy of Sciences.

a)Email: okulov@imp.uran.ru

${ }^{1}$ V. I. Okulov, A. V. Gergert, T. E. Govorkova, A. V. Korolev, A. T. Lonchakov, and L. D. Sabirzyanova, Fiz. Nizk. Temp. 31, 1143 (2005) [Low Temp. Phys. 31, 872 (2005)]; V. I. Okulov, T. E. Govorkova, V. V. Gudkov, I. V. Zhevstovskikh, A. V. Korolev, A. T. Lonchakov,
K. A. Okulova, E. A. Pamyatnykh, and S. Yu. Paranchich, ibid. 33, 282 (2007) [Low Temp. Phys. 33, 207 (2007)].

${ }^{2}$ V. I. Okulov, E. A. Pamyatnykh, and V. P. Silin, Fiz. Nizk. Temp. 37, 1001 (2011) [Low Temp. Phys. 37, 798 (2011)]

${ }^{3}$ V. I. Okulov, A. T. Lonchakov, T. E. Govorkova, K. A. Okulova, S. M. Podgornykh, L. D. Paranchich, and S. Yu. Paranchich, Fiz. Nizk. Temp. 37, 281 (2011) [Low Temp. Phys. 37, 220 (2011)].

${ }^{4}$ V. I. Okulov, V. V. Gudkov, I. V. Zhevstovskikh, A. T. Lonchakov, L. D. Paranchich, and S. Yu. Paranchich, Fiz. Nizk. Temp. 37, 443 (2011) [Low Temp. Phys. 37, 347 (2011)].

${ }^{5}$ V. I. Okulov, E. A. Pamyatnykh, and Yu. I. Zabaznov, Solid State Phenom. 168-169, 489 (2011).

${ }^{6} \mathrm{~V}$. Yu. Irkhin and Yu. P. Irkhin, Electronic Structure, Physical Properties, and Correlation Effects in $d$ - and f-Metals and their Compounds, NITs

"Regulyarnaya i khaoticheskaya dinamika," (Moscow-Izhevsk, 2008) [in Russian].

${ }^{7}$ N. Nagaosa, J. Sinova, S. Onoda, A. H. MacDonald, and N. P. Ong, Rev. Mod. Phys. 82, 1539 (2010).

${ }^{8}$ P. S. Zyryanov and M. I. Klinger, Quantum Theory of Electron Transport Phenomena in Crystalline Semiconductors (Nauka, Moscow, 1976) [in Russian].

${ }^{9}$ P. S. Zyryanov and V. I. Okulov, Fiz. Tverd. Tela 7, 1749 (1965).

${ }^{10}$ A. T. Lonchakov, V. I. Okulov, T. E. Govorkova, M. D. Andriichuk, and L. D. Paranchich, Pis'ma v ZhETF 96, 444 (2012).

Translated by D. H. McNeill 
Low Temperature Physics is copyrighted by the American Institute of Physics (AIP). Redistribution of journal material is subject to the AIP online journal license and/or AIP copyright. For more information, see http://ojps.aip.org/ltp/ltpcr.jsp 